\title{
Positron Annihilation in Corroded Steels St20 and St3S
}

\author{
R. Pietrzak*, W. Smiatek and R. Szatanik \\ Institute of Physics, Opole University, Oleska 48, 45-052 Opole, Poland \\ The paper deals with examining the lifetimes of positrons emitted from \\ ${ }^{22} \mathrm{Na}$ source in corroded St20 and St3S steels. Influence of the method of \\ defecting the sample surfaces on the spectrum of positron lifetimes was in- \\ vestigated. Influence of temperature of initial annealing of samples, plastic \\ deformation, electrolytic hydrogenation and corrosion in $\mathrm{HCl}$ vapors on trap- \\ ping positrons in the examined steels was also determined.
}

PACS numbers: 78.70.Bj, 61.72.Cc, 82.45.Bb

\section{Introduction}

For many years now the phenomenon of positron annihilation has been a commonly applied method for detection of crystalline defects and their interaction with atoms of admixtures in solids.

The positrons emitted by $\beta^{+}$radioactive atomic nuclei penetrate inside the examined sample at a considerable - as regards the atomic scale - distances. On having been thermalized the positron annihilate with an electron of the material. The defects which occur in a solid can trap positrons in an effective way.

Quite a number of investigations confirmed that the positron lifetime depends on the shape and size of open volume defects, whereas the intensity of the component of positron lifetime spectrum that is responsible for annihilation in defects depends on concentration [1-6].

In situations where the defect state of the near-the-surface layer of a solid does not differ in any considerable way from the defect state in deeper layers of the sample, the information obtained from annihilation of positrons emitted from positron source of continuous energy spectrum characterizes the defect state in the bulk of a sample. Sometimes in the investigations of the defect state of the near-the-surface layer to date sources of monoenergetic positrons of regulated energy have been used [7-9]. But if the near-surface defect distribution correlates with the positron implantation range then positrons emitted from the radioactive isotope can be used also to the study of this distribution.

*corresponding author; e-mail: pietr@uni.opole.pl 
We have been applying a similar technique of investigation and interpretation of results ourselves since 1999, while examining positron annihilation in nickel, iron and some steels with a corroded surface [10-14]. We proved that the value of the defect component of positron lifetime spectrum $\left(\tau_{2}\right)$ and its intensity $\left(I_{2}\right)$, for samples with chemically corroded surface, depend on chemical and mechanical conditions, as well as on time of corroding. Moreover, we proved in papers $[10,14]$ that after chemical removal of the corroded layer (thickness of order of $2 \div 3 \mu \mathrm{m}$ ) from sample surfaces, the annihilation parameters such as mean lifetime, value of the higher lifetime component, and also its intensity take on values close to annihilation parameters obtained for non-corroded samples.

The majority of theories dealing with the mechanism and kinetics of corrosion in metals point to the fact that the rate of creating corrosive pitting and their dimensions are influenced by the initial defect state of the surface.

The present paper is devoted to investigating positron lifetime in St20 and St3S steels of different initial defect state of the surface before subjecting them to corrosion in $\mathrm{HCl}$ vapors.

\section{Preparation of the samples}

Positron lifetimes were measured in samples of St3S and St20 steels. The first is a carbon construction steel, of ordinary quality, designed for general use and of the following composition: $0.22 \% \mathrm{C}, 1.1 \% \mathrm{Mn}$ and Si from $0.1 \%$ to $0.35 \%$, whereas St20 steel is a non-alloy steel designed for surface hardening of the following chemical composition: $0.2 \% \mathrm{C}, 0.4 \% \mathrm{Mn}$ and Si from $0.15 \%$ to $0.40 \%$.

The majority of samples were rectangular prisms of $10 \times 10 \times 0.6 \mathrm{~mm}^{3}$. Only the samples that were treated with plastic stretching were $0.2 \mathrm{~mm}$ thick. All the samples were initially cleansed chemically in a water solution of sulphuric and hydrochloric acids and then annealed at given temperatures (ranging from 140 to $\left.850^{\circ} \mathrm{C}\right)$ under lowered pressure $\left(p<10^{-5}\right.$ mbar) during 3 hours and slowly cooled to reach the room temperature at the speed of $1.5 \mathrm{~K} / \mathrm{min}$. This procedure resulted in the fact that following the initial annealing at different temperatures the grain sizes in the samples could differ, as well as those samples could be phase non-uniform, with different contributions of $\alpha$ and $\gamma$ phases, depending on the temperature of annealing. Following both the chemical cleansing of the samples and their annealing at given temperature, the spectra of positron lifetime were measured. Between two samples undergoing the same procedure of cleansing and annealing there was placed a ${ }^{22} \mathrm{Na}$ positron source covered with hostaphan foil.

The source-samples sandwich was placed between two scintillation detectors of fast-slow spectrometer used to measure positron lifetimes. The resolution of the spectrometer (FWHM) amounted to 270 ps. Then some of the samples corroded in vapors or in a $2 \% \mathrm{HCl}$ water solution. The corrosion occurred in a closed vessel, while the samples were placed at the distance of $2 \mathrm{~cm}$ above the free surface of the solution or were dipped in the solution. The corrosion time in the solution 
lasted 1 hour, whereas in vapors - 100 hours. After being corroded, the samples were cleansed in distilled water and dried with a stream of atmospheric air at room temperature; then, the spectra of positron lifetime were measured again in the geometrical system described above. A detailed description of the sample preparation, geometry of measurements of the positron lifetime spectrum can be found in Ref. [14].

A few of the samples were subjected to one-axis stretching for 150 hours before corrosion, which caused a $3 \%$ elongation (plastic deformation). On removing the stretching force, the samples corroded in $\mathrm{HCl}$ vapors for 100 hours, and then — after being dried — were subject to annihilation investigations.

Annealing the samples at various temperatures and their stretching served the purpose of changing the defect state of the near-the-surface layer of the samples, which - later on, due to corroding - formed new defects or broadened the ones that already existed in it.

It has also been known for many years that electrolytic hydrogenation of iron and steel causes new defects to form in the sample [15-17]. We think that hydrogen that penetrates into the metal forms given defects in the near-the-surface layer. Therefore, some samples, after having their surface chemically purified, were electrolytically hydrogenated with $0.1 \mathrm{n}$ water solution of sulphuric acid, where the sample was the cathode and the electrode made of platinum was the anode. Hydrogenation followed at the current density of $20 \mathrm{~mA} / \mathrm{cm}^{2}$ during 1 hour. After being rinsed and dried, the samples corroded in the $\mathrm{HCl}$ solution during 1 hour. Positron lifetimes were examined following chemical cleansing of the surfaces, after hydrogenation and after corrosion.

In order to determine from which corroded part of the sample comes the information on annihilation of positrons (lifetimes and intensities of the spectrum components) on taking measurements of the lifetimes in corroded samples, the corroded layer was removed chemically through immersing the samples in a solution of rust remover (the brand name FOSOL) for a few minutes. Next, after the rinsing and drying procedures positron lifetime spectra were measured again. Before removing the corroded layer and after its removal the samples were weighed. This allowed determining the thickness of the removed layer.

The analyses of spectra were done with the use of LT 9.0 program. The spectra were analyzed through their decomposition into two or three lifetime components. It was found that in all of the cases, the decomposition of the spectrum into three components yields a much higher value of the $\chi^{2}$ coefficient than in the case of its decomposition into two components $\tau_{1}$ and $\tau_{2}$ and intensities $I_{1}$ and $I_{2}$, respectively. It is known that the positron lifetimes in free state in iron amounts to $106 \mathrm{ps}$ when determined by us values of $\tau_{1}$ for the corroded samples were in the limit $140 \div 170 \mathrm{ps}$. From the many-state model of positron annihilation it follows that the value of the shortest component of the positron lifetime spectrum $\tau_{1}$ is always lower than the positron lifetimes in the free state. Analysing the obtained 
values of $\tau_{1}$ and $\tau_{2}$ we can assume that $\tau_{1}$ component is a superposition of lifetimes of positrons which annihilate in defects of small sizes in the near-the-surface layer (vacancies and dislocations), called "defects of type I" further in the text, while $\tau_{2}$ - lifetime of positrons which annihilate in defects of larger sizes (e.g. in multivacancy clusters, microcracks) from now on called "defects of type II". We assume that the concentrations of defects is so high that all the positrons are trapped by defects at rate $K_{1}$ and $K_{2}$, respectively, and annihilate in them after times $\tau_{1}$ and $\tau_{2}$ with the provision that

$$
\begin{aligned}
& K_{1}=\mu_{1} C_{1}, \\
& K_{2}=\mu_{2} C_{2},
\end{aligned}
$$

where $\mu$ and $C$ denote coefficients of the rate of positron trapping and concentrations of defects of both types, respectively. With the assumption of non-occurrence of de-trapping positrons from defects and that $k_{1} \gg 1 / \tau_{\mathrm{f}}$ and $k_{2} \gg 1 / \tau_{\mathrm{f}}$ the intensity of the second component $I_{2}$ is described by the following dependence:

$$
I_{2}=\frac{\mu_{2} C_{2}}{\mu_{1} C_{1}+\mu_{2} C_{2}} .
$$

It follows from the last dependence that the rise in type II defects concentration causes a rise in the intensity of the other component $I_{2}$.

Due to the fact that corrosion processes lead to a rapid formation of "large" defects, we shall only deal with interpreting the changes in the parameters relating to the component of the longer positrons lifetime $\left(\tau_{2}\right.$ and $\left.I_{2}\right)$.

The lifetimes of positrons in defects and coefficients of trapping rate in them are functions of sizes of defects. One can, thus, assume that changes in the value of $\tau_{2}$ component of positron lifetimes spectrum are connected with changes in the size of defects, in which positrons annihilate [5], the changes in intensity $I_{2}$ being related to the changes in their concentration.

\section{Results and interpretation}

Positron lifetime in steels St20 and St3S, prior and following the mechanical, chemical working, as well as corrosion, were determined. Figures 1 and 2 present influence of the temperature of initial annealing of St20 and St3S samples on values of annihilation parameters (second component $\tau_{2}$ and also its intensity $I_{2}$ ) prior to corrosion (A) and following the corrosion (B), as well as chemical removal of corroded layer of thickness of $2.67 \mu \mathrm{m}$ for St20 steel (C).

Initial annealing of steel St20 at higher and higher temperatures causes a decrease in the value of $\tau_{2}$ and its intensity, which - according to the interpretation presented above - means decreasing the sizes of multivacancy clusters. A 100 hour long corroding in $\mathrm{HCl}$ vapors of samples annealed prior to the procedure at $140^{\circ} \mathrm{C}$ does not cause any visible changes in the values of $\tau_{2}$ and $I_{2}$, whereas keeping samples of this steel annealed prior to that in a corrosive atmosphere at $580^{\circ} \mathrm{C}$ does bring about a visible expansion of vacancy clusters (a rise in the value of 

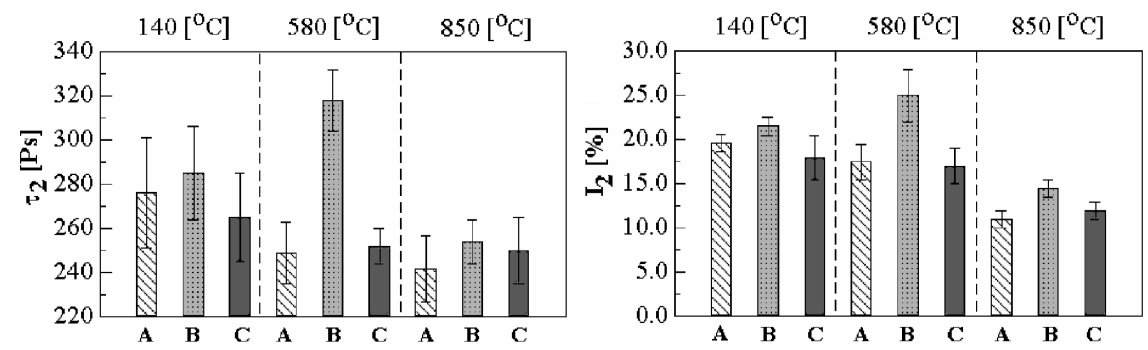

Fig. 1. Influence of temperature of initial annealing on the values of the second positron lifetimes component $\tau_{2}$ and its intensity $I_{2}$ measured for St20 steel: A - for samples annealed at determined temperatures; $\mathrm{B}$ - after $100 \mathrm{~h}$ long corrosion treatment in $\mathrm{HCl}$ vapors; C - after chemical removal of the corroded layer.
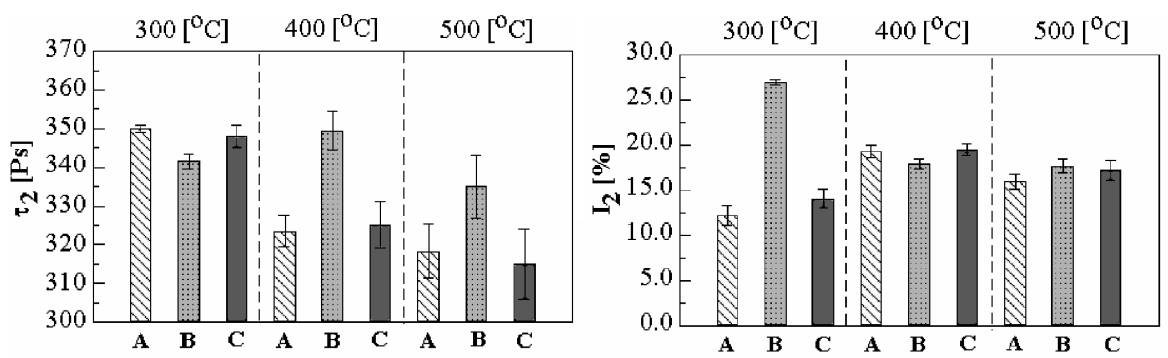

Fig. 2. As in Fig. 1 but for St3S steel.

component $\tau_{2}$ ) and their concentrations (a rise in intensity $I_{2}$ ). A rise in the concentration and sizes of these defects occurs in the close-to-the-surface layer, since after removing the corroded layer of $2.67 \times 10^{-3} \mathrm{~mm}$ thickness the again determined annihilation parameters take the same values as before corrosion.

Initial annealing of samples of steel St20 at $850^{\circ} \mathrm{C}$ causes a considerable decrease in the concentration of type II defects. This takes place, most likely, as a result of a decline of defects of large sizes. Storing of thus prepared samples of steel St20 in a corrosive atmosphere during 100 hours leads to an increase in the concentration of type II defects and their growth. It is worth observing, however, that these changes are far smaller than those in the samples annealed initially at $580^{\circ} \mathrm{C}$.

Initial annealing of samples of steel St3S and subsequent storing them in a corrosive environment causes changes only partially similar to those observed for steel St20 (Fig. 2). Like in the case of the latter, the higher the annealing temperature, the smaller the sizes of type II defects ( $\tau_{2}$ decreases). On the other hand, their concentration alters in a non-monotonic manner along with the rise in the temperature of the initial annealing. Keeping samples of the steel St3S in a corrosive atmosphere results in a very clear rise in the intensity of the other component of the positron lifetime spectrum only for samples annealed at tem- 
perature $300^{\circ} \mathrm{C}$. There follows an increase in the concentration of defects of large sizes (type II). This probably occurs as a result of their decomposition into defects of smaller sizes (a rise in $I_{2}$, decrease in the value of $\tau_{2}$ ). However, in steel St3S samples, initially annealed at 400 or $500^{\circ} \mathrm{C}$, the corrosive processes cause a growth in the near-the-surface type II defects, without forming new ones as the intensity of the other component of the positron lifetimes spectrum $I_{2}$ remains constant within the limits of error, that is on the same level as that before corrosion. After removing the corroded near-the-surface layer, parameters $\tau_{2}$ and $I_{2}$ take, like in steel St20, the same values as those before corrosion.

Through weighing the samples three times, that is before and after corrosion and after the removal of the corroded layer, the thickness of the corroded layer was determined. The thickness of removed layers stayed within the range between $2.67 \mu \mathrm{m}$ and $2.76 \mu \mathrm{m}$. This means that annihilation-related information on concentration of defects of type II $\left(I_{2}\right)$ and sizes of the both types of defects $\left(\tau_{1}, \tau_{2}\right)$ come also from layers of thickness of this order.

Numerous investigations into the processes accompanying corrosion of metals indicate a great role of near-the-surface defects on the kinetics of corrosion. In fact, during the plastic deformation of metals the defects (vacancies, dislocations and multivacancy clusters) are generated in the whole volume of deformed material. These defects most probably appear also in the near-the-surface layer. We conducted investigations into the positron lifetimes in samples that were plastically deformed by one-axis stretching prior to corrosion (Fig. 3). First of all, positron

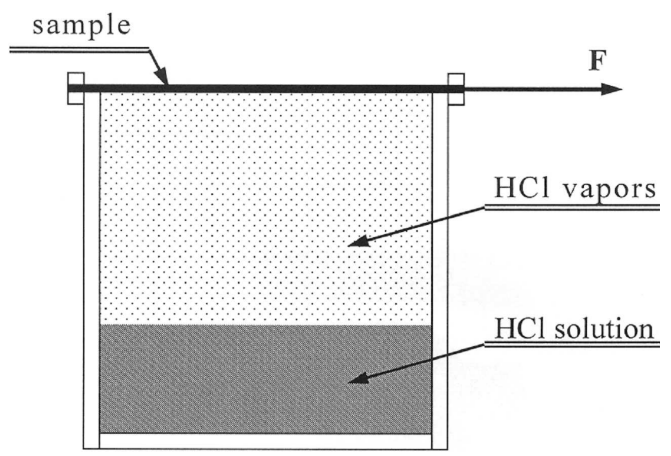

Fig. 3. Scheme of the apparatus to measure the influence of uniaxial stretching on corrosion of steel in $\mathrm{HCl}$ vapors.

lifetimes were measured in samples of St20 steel annealed in vacuum for 3 hours at $850^{\circ} \mathrm{C}$ and cooled to reach room temperature at about $1.3 \mathrm{~K} / \mathrm{min}$ (Fig. 4, bars A). The statistical scatter of results obtained for the steel samples annealed at $850^{\circ} \mathrm{C}$ is fairly big, exceeding measurement-related uncertainties. This is most probably caused by a certain gradient of temperature in the vessel in which the samples were annealed. However, we interpret the results presented in Fig. 4 as relative 



Fig. 4. Influence of one-axis stretching by external forces on the values of positron lifetimes component $\tau_{2}$ and its intensity $I_{2}$ measured for St20 steel: A - for samples annealed at $850^{\circ} \mathrm{C}$; B - after $100 \mathrm{~h}$ long corrosion treatment in $\mathrm{HCl}$ vapors; $\mathrm{C}$ - after $150 \mathrm{~h}$ long stretching in the area of plastic deformations; D - after plastic stress and then $100 \mathrm{~h}$ long corrosion in $\mathrm{HCl}$ vapors; $\mathrm{E}$ - after $100 \mathrm{~h}$ long corrosion in $\mathrm{HCl}$ vapors in a state of plastic stress $\left(\Delta l / l_{0}=3 \%\right)$.

changes in relation to the initial samples (bars A) - for a determined sequence of procedure.

Then, the samples were plastically stretched (relative elongation of the samples amounted to $3 \%$ ) for 3 hours. After removing the external stress, the positron lifetime spectrum was measured again (Fig. 4, bars C) and, next, the samples were corroded in $\mathrm{HCl}$ vapors for 100 hours and the positron lifetime spectrum was again measured (Fig. 4, bars D).

It is surprising to note that the rise in the value of $\tau_{2}$ almost doubled while the value of $I_{2}$ decreased for this component of the spectrum in the case of the sample corroding after prior plastic deformation. It can mean that at a great concentration of the close-to-the-surface multivacancy clusters the chemical processes leading to corrosion cause certain loosening of the crystalline structure and a change of the chemical energy into the kinetic energy of atoms. In this situation the defects become more active, as a result of which they can be transformed or aggregated.

Lifetimes of positrons were also investigated in samples corroding in the state of plastic stretching induced by an external force which caused a $3 \%$ rise in the length in $\mathrm{HCl}$ vapors (Fig. 4, bars $\mathrm{E}$ ). It can be noticed that for samples annealed at $850^{\circ} \mathrm{C}$ and then corroding for 100 hours in $\mathrm{HCl}$ vapors, the value of component $\tau_{2}$ of positron lifetime spectrum remains unchanged within the limits of error, though. Still its intensity grows very markedly. It can be thought, therefore, that during corrosion of $\mathrm{St} 20$ steel in $\mathrm{HCl}$ vapors there form new defects (Fig. 4, bars B) in its near-the-surface layer. As it follows from Fig. 4 (bars C), after stretching force has been removed, the value of component $\tau_{2}$ (linked to defects of type II) remains, within the limits of error, unchanged. Still its intensity $I_{2}$ increases in a visible way.

When a sample was corroding in $\mathrm{HCl}$ vapors for 100 hours in the state of plastic stress $\left(\Delta l / l_{0}=3 \%\right)$ and the lifetimes were measured after corrosion and after removal of the stretching force, the value of the second component of positron 
lifetime spectrum were lower, still the intensity of the other component $I_{2}$ is greater than that in a non-defected sample (Fig. 4, bars E). It can be supposed that in a corroded sample in the state of plastic deformation, concentration of defects about large sizes in the near-the-surface layer is greater than that in a sample deformed earlier but corroding without external stress.

As Pająk et al. proved [15, 16], and also Wu and Jean [17], electrolytic hydrogenation of samples of metals and steel causes, on the one hand, trapping hydrogen atoms in lattice defects, due to which they become inaccessible for positrons, on the other one - causes formation of new defects such as monovacancies, dislocations and multivacancy clusters. We assumed that during electrolytic hydrogenation of samples of steel, there form, in their near-the-surface layers, defects whose presence influences the kinetics and mechanism of corrosion at a later stage. This should be reflected in the annihilation characteristics of the examined samples after corrosion.

Annihilation of positrons was investigated in samples corroded in $\mathrm{HCl}$ solution and vapors and also in ones that were electrolytically hydrogenated in a solution of sulphuric acid before corrosion. However, these samples were taken from a different lot of material from that of the samples discussed above. Hence, the values $\tau_{2}$ and $I_{2}$ are different in their case. The density of hydrogenation current amounted to $20 \mathrm{~mA} / \mathrm{cm}^{2}$, and the hydrogenation lasted 1 hour. As can be seen in Fig. 5, the value of component $\tau_{2}$ for hydrogenated samples remains, within
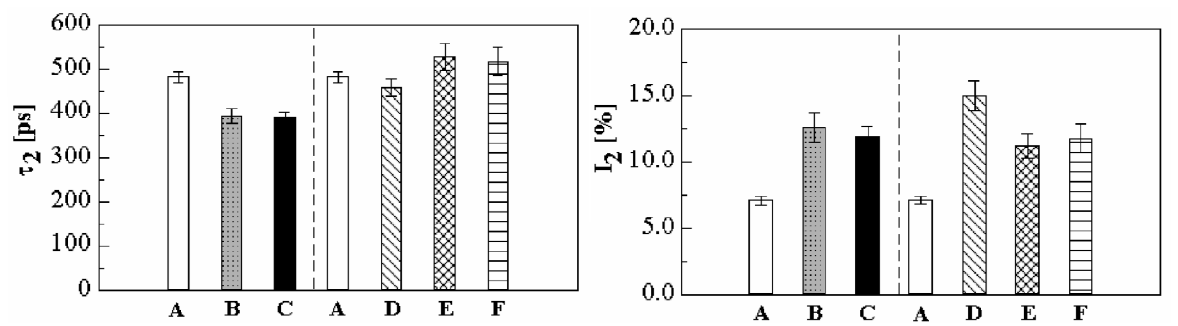

Fig. 5. Influence of electrolytic hydrogenation and conditions of corrosion of St3S steel on the values of positron lifetimes component $\tau_{2}$ and its intensity $I_{2}$ : A - after chemical purification of the surface; $\mathrm{B}$ - after $0.5 \mathrm{~h}$ long corrosion treatment in $2 \% \mathrm{HCl}$ solution; $\mathrm{C}$ - after $1 \mathrm{~h}$ long corrosion treatment in a $2 \% \mathrm{HCl}$ solution; D - after electrolytical hydrogenation; $\mathrm{E}$ - after $0.5 \mathrm{~h}$ long corrosion treatment in a $2 \% \mathrm{HCl}$ solution of a sample hydrogenated earlier; F - after $1 \mathrm{~h}$ long corrosion treatment in a $2 \% \mathrm{HCl}$ solution of a sample hydrogenated earlier.

the limits of error, unchanged, though its intensity $I_{2}$ clearly increased. Still, following corrosion of samples which were hydrogenated earlier, both the component of shorter lifetime $\tau_{1}$ and the "long-life" one, $\tau_{2}$, increase their values. Because there follows a simultaneous lowering in the intensity $I_{2}$ of the other components of positron lifetime spectrum, this can mean that corrosion causes a growth of 
near-the-surface defects through their association, lowering their concentration at the same time.

A full explanation of all the aspects of the results presented hereby requires conducting additional research. Investigations into the problem are currently being carried out and their results will be presented in coming publications.

\section{Conclusions}

On the basis of the investigations carried out the following conclusions can be reached:

1. In St20 and St3S steels the near-the-surface layer can be studied by positron lifetime method.

2. The corroded surface of the investigated steels contains a variety of defects, whose nature and sizes are reflected in positron lifetime spectra.

\section{References}

[1] W. Brandy, H.F. Wuang, Phys. Lett. 27, 700 (1968).

[2] J.K. Mc Kenzie, T.L. Khoo, A.B. Mc Donald, B.T. Mc Kee, Phys. Rev. Lett. 19, 146 (1976).

[3] M.J. Puska, R.M. Nieminen, J. Phys. F, Metal Phys. 13, 333 (1983).

[4] E. Kuramoto, H. Abe, M. Takenaka, F. Hori, Y. Kamimura, M. Kiura, K. Ueno, J. Nucl. Mater. 239, 54 (1996).

[5] Positron Spectroscopy of Solid State, Eds. A. Dupasquier, A.J. Mills Jr., IOS Press, Amsterdam 1995.

[6] P. Hautojarvi, K. Johanson, T. Judin, P. Moser, M. Puska, A. Vehanen, K. Yli-Kaupilla, in: Proc. 5th Int. Conf. on Positron Annihilation, Lake Yamanaka (Japan) 1979, p. 737.

[7] P.J. Schutz, K.G. Lynn, Rev. Mod. Phys. 60, 701 (1988).

[8] P.G. Coleman, Positron Beams and Their Applications, World Sci., Singapore 2000.

[9] K.G. Lynn, in: Positron Solid State Physics, Eds. N. Brandt, A. Dupasquier, North-Holland, Amsterdam 1983, p. 60.

[10] R. Pietrzak, R. Szatanik, M. Szuszkiewicz, Acta Phys. Pol. A 95, 647 (1999).

[11] R. Pietrzak, W. Smiatek, R. Szatanik, M. Szuszkiewicz, Fracture Mechanics of Materials and Structural Integrity 1, 178 (1999).

[12] R. Pietrzak, W. Smiatek, R. Szatanik, M. Szuszkiewicz, in: Proc. 31st Seminar on Positron Annihilation, Jarnottówek (Poland) 1999, Eds. W. Świątkowski, K. Jerie, University of Opole, Opole 2000, p. 123.

[13] R. Pietrzak, R. Szatanik, W. Smiatek, in: Proc. 33rd Polish Seminar on Positron Annihilation, Turawa (Poland) 2001, Ed. K. Jerie, University of Opole, University of Wrocław, Opole 2001, p. 79.

[14] R. Pietrzak, in: Proc. 34th Polish Seminar on Positron Annihilation, Turawa (Poland) 2002, Ed. K. Jerie, University of Opole, University of Wrocław, Opole 2002, p. 61. 
[15] J. Pajạk, R. Pietrzak, M. Szuszkiewicz, Nukleonika 42, 185 (1997).

[16] J. Pająk, R. Szatanik, M. Szuszkiewicz, Acta Phys. Pol. A 95, 637 (1999).

[17] Y.C. Wu, Y.C. Jean, Phys. Status Solidi A 201, 917 (2004). 